\title{
Menopause and postprandial metabolic and inflammatory responses: a cross-sectional study exploring associations with sleep, physical activity and habitual diet
}

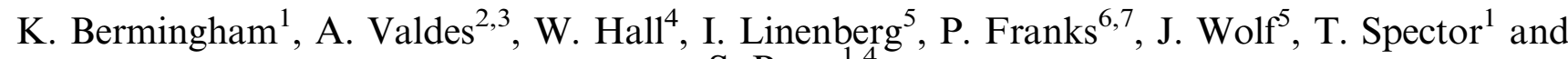 \\ S. Berry 1,4 \\ ${ }^{1}$ Department of Twin Research and Genetic Epidemiology, King's College London, London, UK, \\ ${ }^{2}$ School of Medicine, University of Nottingham, Nottingham, UK, \\ ${ }^{3}$ Nottingham NIHR Biomedical Research Centre, Nottingham, UK, \\ ${ }^{4}$ Department of Nutritional Sciences, King's College London, London, UK, \\ ${ }^{5}$ Zoe Limited, London, UK, \\ ${ }^{6}$ Department of Clinical Sciences, Lund University, Sweden and \\ ${ }^{7}$ Department of Nutrition, Harvard Chan School of Public Health, Boston, USA
}

Cardiometabolic disease risk increases after menopause, related to the depletion of sex steroid hormones ${ }^{(1)}$. Extensive evidence shows that menopausal status contributes to unfavourable fasting metabolic profiles ${ }^{(2)}$, but to date the role of menopause status on postprandial metabolic responses to food are lacking. The PREDICT 1 study is the largest study to date to measure detailed postprandial responses using standardized test meal models. The aim of this study was to understand the impact of menopause on postprandial responses in glucose, triglycerides, insulin and glycoprotein acetylation (GlycA; a marker of inflammation).

Self-reported pre- (pre-M, $n=366$ ), peri- (peri-M, $n=55$ ) and post-menopausal (post-M, $n=207$ ) females aged 18-65 years were identified from the PREDICT 1 study, a multi-centre dietary intervention study of 1,002 healthy individuals from the UK (NCT03479866). Postprandial (0-6 h) metabolic responses to a standardised metabolic challenge meal following an overnight fast were tested including glucose, insulin, triglycerides and GlycA (an inflammation marker) concentrations. Differential responses between groups were analysed for glucose ${ }_{\mathrm{iAUC} 0-2 \mathrm{~h}}$, insulin $_{\mathrm{iAUC0}-2 \mathrm{~h}}$, triglyceride ${ }_{\mathrm{iAUC0}-6 \mathrm{~h}}$ and $\mathrm{Glyc}_{360 \mathrm{rise}}$.

Age-adjusted residuals were used from pre-M and post-M age matched females $(n=150$, age range $47-56$ years). Linear regression modelling determined the independent impact of menopausal status on postprandial response and investigated the association between postprandial measures (glucose ${ }_{i A U C 0-2 h}$, insulin ${ }_{i A U C 0-2 h}$, triglyceride ${ }_{i A U C 0-6 h}$ and GlycA ${ }_{360 \text { rise }}$ ) and exposures (BMI, sleep, physical activity, diet quality, fasting triglyceride (TG), fasting glucose, hormonal replacement therapy (HRT) and age since menopause). $R^{2}$ values were ranked.

Post-menopausal status was associated with significantly poorer sleep quality, greater visceral fat mass and fasting blood measures (glucose, triglycerides and GlycA), $\mathrm{p}<0.05$ for all. Postprandial responses for glucose $_{\mathrm{iAUC0}-2 \mathrm{~h}}$, insulin $_{\mathrm{iAUC}-2 \mathrm{~h}}$, triglyceride $_{\mathrm{AAUC} 0-6 \mathrm{~h}}$ and Glyc $\mathrm{A}_{360 \text { rise }}$ were significantly higher in post-M females by $41,5,33,700 \%$ respectively. Postprandial peak glucose (30 min rise) was significantly higher in pre-M vs post-M females independent of age ( $\beta-0.66$, SE 0.26$)$. When exposures (listed above) associated with postprandial responses (glucose, triglycerides, insulin and GlycA) were assessed separately in pre-M and post-M females, HRT exerted protective postprandial effects in post-M females and fasting TG were in the top 3 ranked determinants for all postprandial measures. However, the proportion of variance explained by each exposure was different between pre-M and post-M females across postprandial measures, such that post-M females had relatively greater influence of fasting TG for Glyc $\mathrm{A}_{360 \text { rise, }} \mathrm{BMI}_{\mathrm{M}}$ for insulin $_{\mathrm{iAUC0}-2 \mathrm{~h}}$, sleep for glucose $\mathrm{iAUC0}_{\mathrm{A}-2 \mathrm{~h}}$ and physical activity for triglyceride $\mathrm{iAUC0}_{\mathrm{A} \text {-6h. }}$. In contrast diet played a larger role in pre-M females for Glyc $\mathrm{A}_{360 \text { rise }}$ and triglyceride $\mathrm{iAUC0}_{\mathrm{A}} \mathrm{h}$.

In the largest postprandial study performed to date we demonstrate unfavourable postprandial responses are associated with menopause. Metabolic and lifestyle factors including diet, sleep and physical activity contribute differentially to postprandial metabolic response in pre-M and post-M females. This study shows the potential to provide stratified advice to reduce the adverse metabolic effects associated with menopause.

\section{References}

1. Stachowiak G, Pertyński T \& Pertyńska-Marczewska M (2015) Menopause review 14(1), 59.

2. Janssen I, Powell LH, Crawford S et al. (2008) Arch Intern Med 168(14), 1568-75. 